\title{
Sexual coevolution of spermatophore envelopes and female genital traits in butterflies: Evidence of male coercion?
}

Signa are sclerotized structures located on the inner wall of the corpus bursa of female Lepidoptera whose main function is tearing open spermatophores. The sexually antagonistic coevolution (SAC) hypothesis proposes that the thickness of spermatophore envelopes has driven the evolution of the female's signa; this idea is based in the fact that in many lepidopterans female sexual receptivity is at least partially controlled by the volume of ejaculate remaining in the corpus bursa. According to the SAC hypothesis, males evolved thick spermatophore envelopes to delay the post-mating recovery of female sexual receptivity thus reducing sperm competition; in response, females evolved signa for breaking spermatophore envelopes faster, gaining access to the resources contained in them and reducing their intermating intervals; the evolution of signa, in turn, favored the evolution of even thicker spermatophore envelopes, and so on. We tested two predictions of the SAC hypothesis with comparative data on the thickness of spermatophore envelopes of eleven species of Heliconiinae butterflies. The first prediction is that the spermatophore envelopes of polyandrous species with signa will be thicker than those of monandrous species without signa. In agreement with this prediction, we found that the spermatophore envelopes of a polyandrous Heliconius species with signa are thicker than those of two monandrous Heliconius species without signa. The second prediction is that in some species with signa males could enforce monandry in females by evolving "very thick" spermatophore envelopes, in these species we predict that their spermatophore envelopes will be thicker than those of their closer polyandrous relatives with signa. In agreement with this prediction, we found that in two out of three comparisons, spermatophore envelopes of monandrous species with signa have thicker spermatophore envelopes than their closer polyandrous relatives with signa. Thus, our results support the idea that selective pressures arising from sexually antagonistic interactions have been important in the evolution of spermatophore envelopes, female signa and female mating patterns. 


\section{INTRODUCTION}

2 During sexual interactions males and females exert selection pressures on the opposite sex that

3 can produce reciprocal adaptations in a process known as sexual coevolution (Parker, 1979;

4 Eberhard, 1985, 1996; Holland \& Rice, 1998). There is increasing evidence that sexual

5 coevolution is responsible for the evolution of many structural and functional aspects of animal

6 genitalia (Eberhard, 1985, 1996, 2010; Hosken \& Stockley, 2004; Arnqvist \& Rowe, 2005;

7 Minder, Hosken \& Ward, 2005; Brennan et al., 2007; Sánchez, Hernández-Baños \& Cordero,

8 2011; Breed, Leigh \& Speight, 2013; Burns, Hedin \& Shultz, 2013; Yassin \& Orgogozo, 2013).

9 For example, in species in which females increase their fitness by mating with multiple mates,

10 males could evolve genital structures for damaging female genitalia if this damage decreases

11 female mating rate; these structures, in turn, could select for protective genital structures in

12 females. In the Drosphila melanogaster species subgroup evidence indicates that females have

13 coevolved genital structures that protect them from damage by male genital structures (Yassin \&

14 Orgogozo, 2013). In other species, also exhibiting adaptive polyandry, females could evolve

15 genital traits that allow them to discriminate among males of different quality during copulation;

16 these traits could select for elaborate male intromittent genitalia for internal stimulation of the

17 females (Eberhard, 1985). Evidence suggests that the extremely complex vaginal morphology of

18 waterfowl species coevolved with the long and complex male phallus as a cryptic choice

19 mechanism (Brennan et al., 2007).

20 In the particular case of Lepidoptera, in a previous paper we presented comparative

21 evidence supporting the hypothesis that the sclerotized structures called signa, present in the

22 inner genitalia of females from many species, are a product of antagonistic coevolution with

23 males (Sánchez, Hernández-Baños \& Cordero, 2011). The signa are located on the inner wall of

24 the female's corpus bursa-the bag-like receptacle where males deposit a spermatophore during 
25 copulation-and are used for breaking the spermatophore envelope and gain access to its contents 26 (Hinton, 1964; Galicia, Sánchez \& Cordero, 2008). Our sexually antagonistic coevolution (SAC)

27 hypothesis proposes that, since in many polyandrous Lepidoptera the length of time a female

28 remains sexually unreceptive after mating is directly related to the amount of ejaculate remaining

29 in her corpus bursa (Sugawara, 1979; Drummond, 1984; Wiklund, 2003; Wedell, 2005), sperm

30 competition selects for males that transfer spermatophores with thick envelopes that take more

31 time to be broken and thus delay female remating beyond her optimum time (Drummond, 1984;

32 Cordero, 2005; Fig. 1). Thick spermatophore envelopes, in turn, select for signa that allow

33 females faster breaking of the envelopes, thus reducing intermating intervals (Cordero, 2005; Fig.

34 1). Our previous comparative analysis supported the prediction from the SAC hypothesis that

35 signa tend to be present mainly in polyandrous species, and suggested that polyandry and signa

36 are plesiomorphic in the Lepidoptera (Sánchez, Hernández-Baños \& Cordero, 2011). The SAC

37 hypothesis also predicts that when monandry is selected for in females, the resulting

38 disappearance of sperm competition favors the evolution of thinner spermatophore envelopes

39 (because they are less expensive to produce) and, in consequence, the loss of signa in females.

40 Our previous study also found support for this prediction, because in several groups (including

41 the pupal mating Heliconius species) the evolution of monandry was accompanied by the loss of

42 signa (Sánchez, Hernández-Baños \& Cordero, 2011). However, in some cases monandry could be

43 imposed by males on females (i.e., could be maladaptive for females) by evolving even thicker

44 spermatophore envelopes in response to the evolution of signa (an analogous effect has been

45 proposed for Heliconius antiaphrodisiacs; Estrada et al., 2011). In this case, the SAC hypothesis

46 predicts the evolution of thicker spermatophore envelopes in monandrous species with signa than

47 in polyandrous species. 
49 spermatophore envelope and presence of signa in species differing in female mating patterns have

50 not been tested. In this respect, the only relevant observations we are aware of are those reported

51 by Matsumoto and Suzuki in a paper on mating plugs in six genera of Papilionidae (Matsumoto

$52 \&$ Suzuki, 1995). We have discussed these data in detail in previous publications (Cordero, 2005;

53 Sánchez, Hernández-Baños \& Cordero, 2011). Briefly, Matsumoto and Suzuki's results support

54 predictions of the SAC hypothesis: monandrous genera are characterized by an absence of thick

55 spermatophore envelopes ("capsule" in their terminology) and a lack of signum; moderately

56 polyandrous species have a "relatively thick" spermatophore envelope and a "small" signum;

57 whereas more polyandrous genera have a "thick" spermatophore envelope and a well developed

58 signum (Matsumoto \& Suzuki, 1995). The agreement of Matsumoto and Suzuki's data with the

59 SAC hypothesis is persuasive, but studies specifically designed to test the predicted relationship

60 between the thickness of spermatophore envelopes and signa are necessary. In this report, we use

61 data on the thickness of spermatophore envelopes of eleven species of butterflies varying in

62 presence of signa and in female mating pattern (Fig. 2A) to test two predictions of the SAC

63 hypothesis. First, we tested the prediction that spermatophore envelopes of polyandrous species

64 with signa are thicker than those of monandrous species without signa (T1 $\rightarrow$ T2 in Fig. 1).

65 Second, we tested the prediction that spermatophore envelopes of monandrous species with signa

66 have thicker spermatophore envelopes than their closer polyandrous relatives with signa; as

67 explained above, the rationale behind this prediction is that in monandrous species with signa

68 monandry is enforced by males via the (co)evolution of "very thick" spermatophore envelopes

$69 \quad(\mathrm{~T} 2 \rightarrow \mathrm{T} 4$ in Fig. 1).

\section{MATERIALS AND METHODS}


71 We collected females from eleven species of the subfamily Heliconiinae (Nymphalidae) (Luis-

72 Martínez, Llorente-Bousquets \& Vargas-Fernández, 2003; Table 1); specimens were captured

73 under a scientific collector permit granted to the second author by the Mexican Secretaría de

74 Medio Ambiente y Recursos Naturales (FAUT-0237). These species were selected to test the

75 predictions mentioned in the introduction on the basis of findings from our previous research

76 (Sánchez, Hernández-Baños \& Cordero, 2011). Information about the absence/presence of signa

77 was obtained from Brown (1981) and confirmed upon dissection. For most species, we used

78 published data about female mating pattern estimated from spermatophore counts in field

79 collected females (Heliconius spp.: Ehrlich \& Ehrlich (1978) and Walters et al. (2012); Eueides

80 spp., Dryadula phaetusa, Dryas iulia, Philaethria diatonica and Dione juno: Ehrlich \& Ehrlich

81 (1978); Agraulis vanillae: Drummond (1984); Dione moneta: data from females collected by VS

82 in the Pedregal de San Angel ecological preserve, located in the main campus of the Universidad

83 Nacional Autónoma de México in southern Mexico City, these females were different from those

84 used for measuring thickness of spermatophore envelopes). Most females were collected in

85 different locations in the state of Veracruz, Mexico. Females were netted, euthanized, and their

86 abdomens preserved in vials with $70 \%$ ethanol until dissection.

87 In the laboratory, the corpus bursae were dissected under a dissection microscope

88 (Olympus SZH10) and only corpus bursae containing complete spermatophores were cut in

89 transversal sections that allowed us measuring the thickness of spermatophore envelopes.

90 (Several females provided no data because they did not contain spermatophores or because the

91 spermatophores they contained were partially or completely digested.) To obtain cross sections of

92 spermatophore envelopes, the corpus bursae containing intact spermatophores were processed in

93 the following sequence: (1) they were left in Bouin fixative solution for $24 \mathrm{~h}$; (2) they were

94 dehydrated in progressively higher concentrations of alcohol (from $50 \%$ to $100 \%$, leaving the 
corpus bursae $1 \mathrm{~h}$ in each concentration); (3) they were left in a 1:1 mixture of Paraplast ${ }^{\circledR}$ tissue

96

97

98

99

100

101

102

103

104

105

106

107

108

109

110 $1111)$

112

113

114

115

116

117

118

embedding media and HistoChoice ${ }^{\circledR}$ clearing agent for $24 \mathrm{~h}$ in an oven at $60 \mathrm{C}^{\circ}$; (4) they were

left in Paraplast ${ }^{\circledR}$ tissue embedding media for $24 \mathrm{~h}$ in an oven at $60 \mathrm{C}^{\circ}$; (5) blocks of Paraplast $\AA$

containing one corpus bursa were elaborated; (6) the whole corpus bursae containing intact

spermatophores were cut transversally in $20 \mu \mathrm{m}$ thick sections with an advanced precision rotary microtome (MD00030); (7) the sections were placed in glass slides, stained with methylene blue, and permanent preparations made using Cytoseal Mounting Medium. Photographs of these preparations were taken under the microscope (Olympus BX-51) with a digital camera (Olympus C-5050), and the thickness of spermatophore envelopes measured in the photographs of the sections with the UTHSCSA ImageTool for Windows version 3.00 software. In each photograph we traced an imaginary cross centered in the middle point of the section and measured the thickness of the spermatophore envelope at each of the four intersection points between the cross and the spermatophore section. The number of spermatophores used per species varied between 2 and 7 (total number of spermatophores studied $=43$ ); the total number of measurements of envelope thickness per spermatophore varied between 55 and 413 (about half of the sample had between 150 and 250 sections measured), mainly due to differences in spermatophore size (Table

The prediction that spermatophore envelopes of polyandrous species with signa are thicker than those of monandrous species without signa was tested by comparing three species of Heliconius, two belonging to the monandrous clade without signa (H. hortense and $H$. charithonia) and the other to the polyandrous clade with signa (H. ismenius) (Beltrán et al., 2007; Fig. 2A). The prediction that spermatophore envelopes of monandrous species with signa are thicker than those of their polyandrous relatives with signa was tested in three independent comparisons (Fig. 2A): (a) polyandrous Eueides aliphera vs. monandrous E. isabella; (b) 
119 polyandrous Dryadula phaetusa + Dryas iulia vs. monandrous Philaethria diatonica; and (c)

120 polyandrous Agraulis vanilla vs. monandrous Dione juno + D. moneta.

\section{RESULTS AND DISCUSSION}

\section{Are the spermatophore envelopes of polyandrous species with signa thicker than those of} 123 monandrous species without signa?

124 The spermatophore envelopes of the polyandrous species with signa (H. ismenius) were thicker

125 than those of the monandrous species lacking signa (H. hortense and H. charithonia) (Kruskal-

126 Wallis ANOVA, $H_{2,12}=8.33, p=0.016$; Fig. $2 \mathrm{~B}$ ). This result is in agreement with the SAC

127 hypothesis that proposes that polyandry selects for males that produce thicker spermatophore

128 envelopes to delay female remating, and that, in response, females evolved signa that allowed

129 them to increase the rate of spermatophore digestion, thus increasing their remating rate

130 (Cordero, 2005; Sánchez, Hernández-Baños \& Cordero, 2011). There were also differences in

131 spermatophore envelope thickness between the two monandrous species (Fig. 2B). Walters and

132 colleagues found that in large samples of some pupal mating monandrous Heliconius species

133 there is a very small proportion of double mated females (Walters et al., 2012); it would be

134 interesting to study large samples of $H$. hortense and H. charithonia to see if some females mate

135 more than once and, in case they do, if the proportion of multiple mated females is larger in $H$.

136 hortense, as would predict the SAC hypothesis.

137 Are spermatophore envelopes of monandrous species with signa thicker than those of their

138 polyandrous relatives with signa? 
139 In two of the three groups compared, the envelopes of the spermatophores received by

140 monandrous females with signa were thicker than those of polyandrous species with signa

141 (Eueides species [Fig. 2C]: Mann-Whitney Test, $U=0, p=0.006$; Dryadula/ Dryas/ Philaethria

142 [Fig. 2D]: Kruskal-Wallis ANOVA, $H_{2,11}=6.91, p=0.032$ ). These results agree with expectations

143 from the SAC hypothesis, that predicts perpetual coevolution between male and female traits and,

144 therefore, considers the possibility of finding instances in which the interests of one of the sexes

145 (males in the present case) prevail over those of the opposite sex (females in the present case), as

146 would be the situation depicted in time 4 of Fig. 1. However, although these results are consistent

147 with the prediction, they do not prove that in E. isabella and P. diatonica monandry is imposed

148 by males and, therefore, maladaptive for females. To test this, it is necessary to show that females

149 of these two species do not remate due to the time taken to break and digest the spermatophore,

150 and that female fitness decreased when they lost the ability to remate due to the evolution of

151 thicker spermatophore envelopes.

152

On the other hand, the third comparison (Fig. 2E) does not support the prediction: the

153 thinner spermatophore envelopes were present in one of the monandrous species (Dione moneta),

154 while the other (D. juno) had spermatophore envelopes as thick as those of the polyandrous

155 species (Agraulis vanillae) (Kruskal-Wallis ANOVA, $H_{2,9}=6.23, p=0.044$ ). An hypothesis to

156 explain this case is that selection favored monandry in female D. moneta, which, in turn, favored

157 the evolution of thin spermatophore envelopes. However, if the reduction in envelope thickness

158 evolves gradually, the decrease in signa size and/or in the size of the spines covering the signa

159 (see next paragraph and Fig. 3) also could be gradual, and the presence of signa and a relatively

160 thin spermatophore envelope could be expected as a transitory evolutionary state. It is interesting

161 to note that, although thinner when compared with $D$. juno and A. vanillae, the spermatophore 
162 163 signa (Fig. 2B).

164

165

166

167

168

169

170

171

172

173

174

envelopes of $D$. moneta are thicker than those of the two monandrous Heliconius species without

A final observation is consistent with the hypothesis of antagonistic coevolution between signa traits and spermatophore envelopes: In the polyandrous $H$. ismenius and the monandrous Eueides isabella, females have two signa shaped like long and thin plates covered with small spines (this general structure is present, with variants, in most species included in this paper), and previous observations indicate that these small spines help breaking open the spermatophore envelope (Galicia, Sánchez \& Cordero, 2008). When we compared the thickness of the spermatophore envelopes with the average length of the spines covering the signa we found a good match between these two measures (Fig. 3). As the SAC hypothesis would predict, the spines are longer in the species with thicker spermatophore envelopes (E. isabella) and in both species they are of a length similar to the thickness of the spermatophore envelopes produced by males of its own species.

\section{CONCLUSIONS}

176 In general terms, most of the comparisons presented in this paper are consistent with the idea that

177 sexually antagonistic selective pressures have been important forces in the evolution of female

178 mating patterns, signa and spermatophore envelope thickness in heliconiinae butterflies

179 (Cordero, 2005; Sánchez, Hernández-Baños \& Cordero, 2011; Fig. 1): (a) The spermatophore

180 envelopes of a polyandrous species with signa are thicker than those of two monandrous species

181 without signa (Figs. 2B); (b) in two out of three cases, males from monandrous species with

182 signa produced thicker spermatophore envelopes than related polyandrous species with signa

183 (Fig. 2C, 2D); and (c) in two species the length of the spines covering the signa matched the 
184 thickness of the spermatophore envelopes produced by males of its own species (Fig. 3). On the

185 other hand, one of the comparisons did not fit the prediction (Fig. 2E), and further studies are

186 necessary to test if monandry is imposed by males in E. isabella and P. diatonica. When we

187 consider that, at least in some species, signa could accomplish different or additional functions to

188 spermatophore tearing (for example, protection from spines in male genitalia; Galicia, Sánchez

$189 \&$ Cordero, 2008; Cordero, 2010), it is not surprising that not all variation in the presence of

190 signa and in spermatophore envelope thickness could be explained by the SAC hypothesis.

191 Future comparative and functional studies are necessary to fully understand the evolution of these 192 traits.

\section{REFERENCES}

194 Arnqvist G, Rowe L. 2005. Sexual Conflict. Princeton University Press, Princeton.

195 Beltrán M, Jiggins CD, Brower AVZ, Bermingham E \& Mallet J. 2007. Do pollen feeding, pupal 196 mating and larval gregariousness have a single origin in Heliconius butterflies?: Inferences from 197 multilocus DNA sequence data. Biological Journal of the Linnean Society 92: 221-239.

198 Breed WC, Leigh CM \& Speight N. 2013. Coevolution of the male and female reproductive 199 tracts in an old endemic murine rodent of Australia. Journal of Zoology 289: 94-100.

200 Brennan PLR, Prum RO, MacCracken KG, Sorenson MD \& Birkhead TR. 2007. Coevolution of 201 male and female genital morphology in waterfowl. PLOS ONE 2(5): e418.

202 Brown KS. 1981. The biology of Heliconius and related genera. Annual Review of Entomology 203 26: 427-456. 
204 Burns MM, Hedin M \& Shultz JW. 2013. Comparative analyses of reproductive structures in

205 harvestmen (Opiliones) reveal multiple transitions from courtship to precopulatory antagonism.

206 PLOS ONE 8(6): e66767.

207 Cordero C. 2005. The evolution of signa in female Lepidoptera: natural and sexual selection

208 hypotheses. Journal of Theoretical Biology 232: 443-449.

209 Cordero C. 2010. On the function of cornuti, sclerotized structures of the endophallus of

210 Lepidoptera. Genetica 138: 27-35.

211 Drummond III BA. 1984. Multiple mating and sperm competition in the Lepidoptera. In: Smith

212 RL, Editor. Sperm Competition and the Evolution of Animal Mating Systems, pp. 291-371.

213 Academic Press.

214 Eberhard WG. 1985. Sexual Selection and Animal Genitalia. Harvard University Press.

215 Eberhard WG. 1996. Female Control: Sexual Selection by Cryptic Female Choice. Princeton

216 University Press.

217 Eberhard WG. 2010. Evolution of genitalia: theories, evidence, and new directions. Genetica 218 138: 5-18.

219 Ehrlich AH, Ehrlich PR. 1978. Reproductive strategies in the butterflies: I. Mating frequency, 220 plugging, and egg number. Journal of the Kansas Entomological Society 51: 666-697.

221 Estrada C, Schulz S, Yildizhan S, Gilbert LE. 2011. Sexual selection drives the evolution of 222 antiaphrodisiac pheromones in butterflies. Evolution 65: 2843-2854.

223 Galicia I, Sanchez V, Cordero C. 2008. On the function of signa, a genital trait of female

224 Lepidoptera. Annals of the Entomological Society of America 101: 786-793. 
225 Hinton HE. 1964. Sperm transfer in insects and the evolution of haemocelic insemination. In:

226 Highnam KC, Editor. Insect Reproduction, pp 95-107. Symposium of the Royal Entomological 227 Society of London.

228 Holland B, Rice WR. 1998. Chase-away sexual selection: antagonistic seduction versus 229 resistance. Evolution 52: 1-7.

230 Hosken DJ, Stockley P. 2004. Sexual selection and genital evolution. Trends in Ecology and 231 Evolution 19: 87-93.

232 Lincango P, Fernández G, Baixeras J. 2013. Microstructure and diversity of the bursa copulatrix 233 wall in Tortricidae (Lepidoptera). Arthropod Structure and Development 42: 247-256.

234 Luis-Martínez A, Llorente-Bousquets JE, Vargas-Fernández I. 2003. Nymphalidae de México I 235 (Danainae, Apaturinae, Biblidinae y Heliconiinae): Distribución Geográfica e Ilustración. 236 UNAM/CONABIO.

237 Matsumoto K, Suzuki N. 1995. The nature of mating plugs and the probability of reinsemination 238 in Japanese Papilionidae. In: Scriber JM, Tsubaki Y, Lederhouse RC, Editors. Swallowtail 239 Butterflies: Their Ecology and Evolutionary Biology, pp. 145-154. Scientific Publishers.

240 Minder AM, Hosken DJ, Ward PI. 2005. Co-evolution of male and female reproductive

241 characters across the Scatophagidae (Diptera). Journal of Evolutionary Biology 18: 60-69.

242 Parker GA. 1979. Sexual selection and sexual conflict. In: Blum MS, Blum N, Editors. Sexual

243 Selection and Reproductive Competition in Insects, pp. 123-166. Academic Press.

244 Sánchez V, Hernández-Baños BE, Cordero C. 2011. The evolution of a female genital trait widely

245 distributed in Lepidoptera: comparative evidence for an effect of sexual selection. PLoS ONE

246 6(8): e22642. doi:10.1371/journal.pone.0022642. 
247 Sugawara T. 1979. Stretch reception in the bursa copulatrix of the butterfly Pieris rapae

248 crucivora, and its role in behavior. Journal of Insect Physiology A 130:191-199.

249 Walters JR, Stafford C, Hardcastle TJ, Jiggins CD. 2012. Evaluating female remating in light of 250 spermatophore degradation in Heliconius butterflies: pupal-mating monandry vs. adult-mating

251 polyandry. Ecological Entomology 37: 257-268.

252 Wedell N. 2005. Female receptivity in butterflies and moths. Journal of Experimental Biology 253 208: 3433-3440.

254 Wiklund C. 2003. Sexual selection and the evolution of butterfly mating systems. In: Boggs CR, 255 Watt WB, Ehrlich PR, Editors. Butterflies: Ecology and Evolution Taking Flight, pp. 67-90.

256 University of Chicago Press.

257 Yassin A, Orgogozo V. 2013. Coevolution between male and female genitalia in the Drosophila 258 melanogaster species subgroup. PLOS ONE 8(2): e57158. doi:10.1371/journal.pone.0057158. 


\section{Figure 1}

Sexually antagonistic coevolution hypothesis of the evolution of spermatophore envelope thickness and signa in Lepidoptera.

Schematic representation of the Sexually Antagonistic Coevolution hypothesis for the coevolution of spermatophore envelopes and signa in Lepidoptera. Arrows represent selective pressures. 


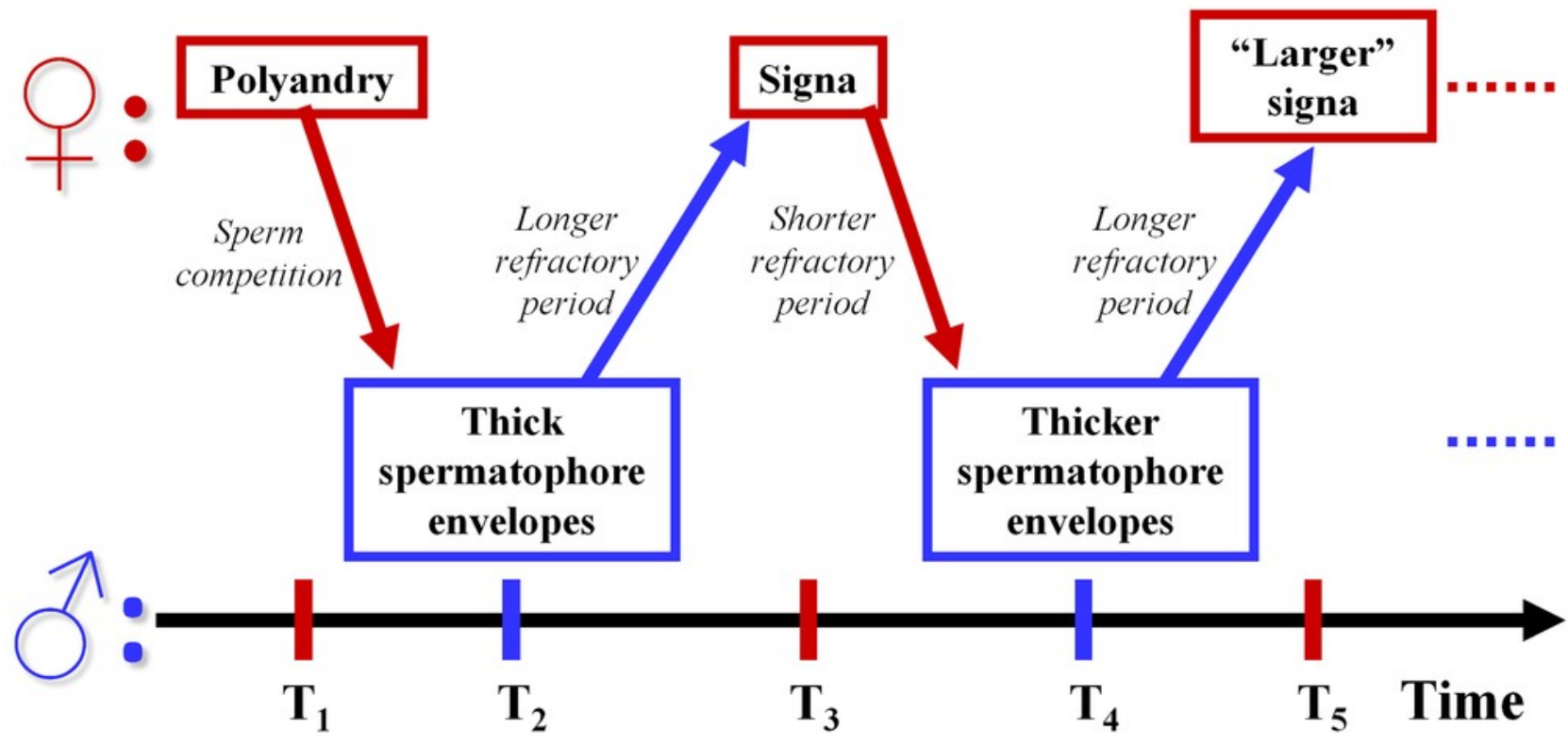




\section{Figure 2}

Comparative tests of the sexually antagonistic coevolution hypothesis (SAC) of the evolution of spermatophore envelope thickness in butterflies.

(A) Phylogenetic relationships between the eleven butterfly species included in the comparisons (this figure is part of the phylogenetic supertree used in the comparative study of Sánchez, Hernández-Baños \& Cordero, 2011). (B) Comparison of spermatophore envelope thickness between one polyandrous species with signa and two monandrous species without signa. As predicted by the SAC, the polyandrous species with signa has thicker envelopes than the monandrous species without signa. (C-E) Three comparisons of spermatophore envelope thickness between polyandrous species with signa and monandrous species with signa. As predicted by the SAC, in comparisons $A$ and $B$ the monandrous species with signa has thicker envelopes than polyandrous species with signa; this pattern was not observed in case C. 


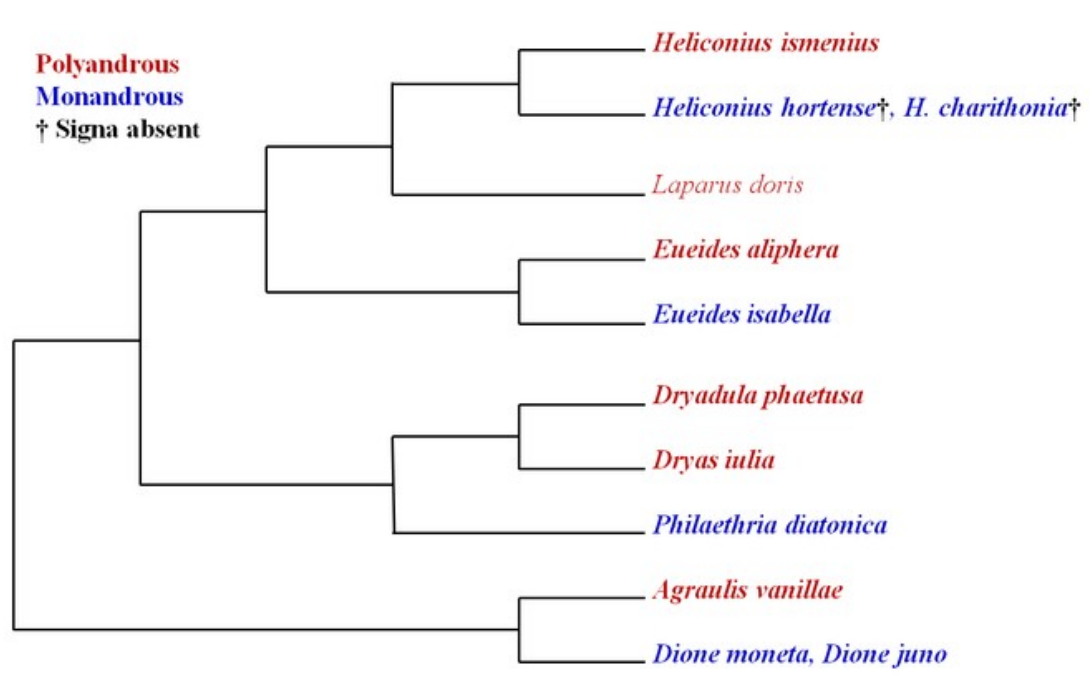

\section{Spermatophore envelope thickness $(\mathrm{mm})$ (average \pm 0.95 confidence intervals):}
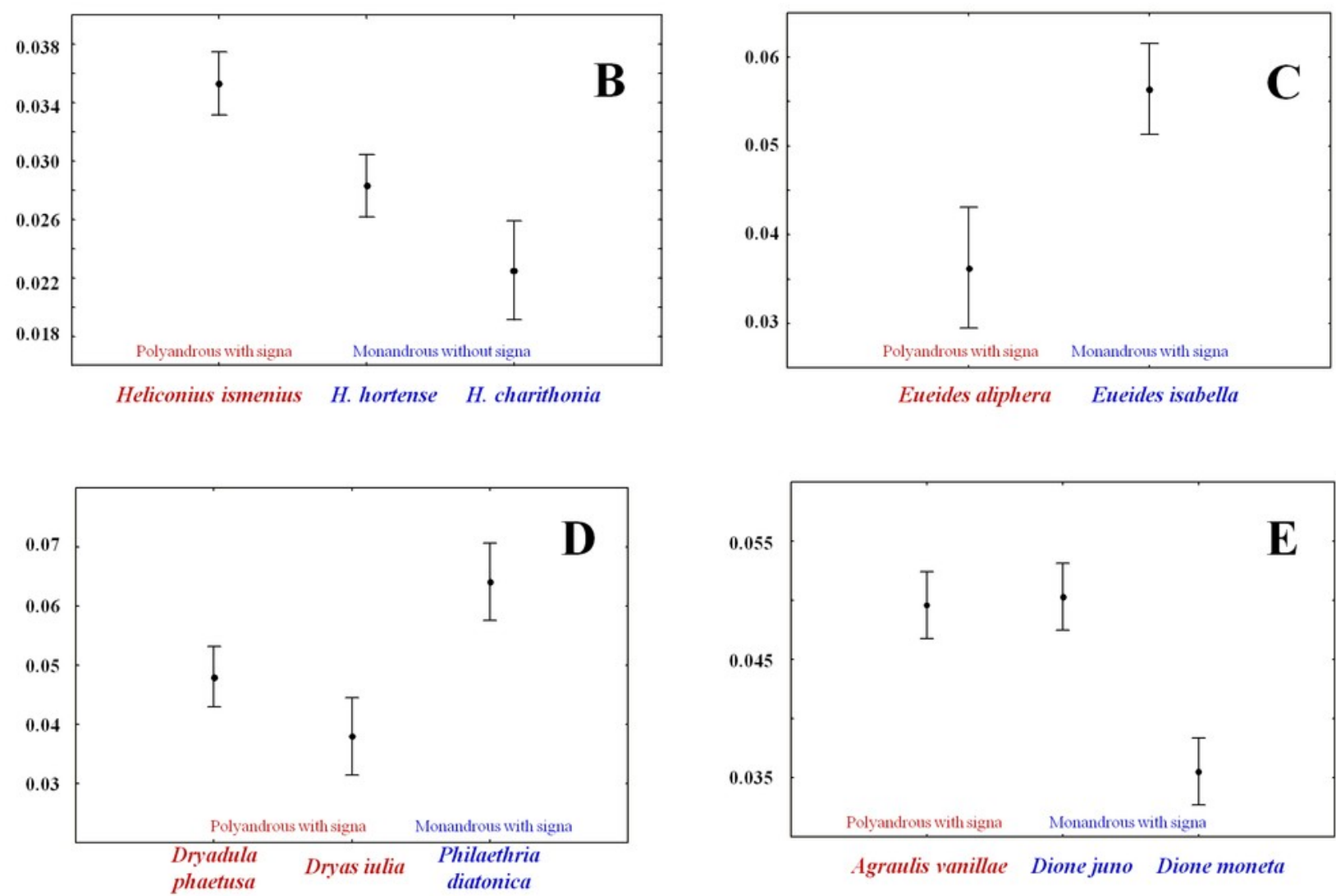


\section{Figure 3}

The length of the spines covering the signa correlates with spermatophore envelope thickness.

(A) Comparisons between the thickness of spermatophore envelopes and the length of the spines that cover the signa in two species selected for producing thick spermatophore envelopes, the polyandrous Heliconius ismenius and the monandrous Eueides isabella. (B) Section of signum covered with spines next to a section of the spermatophore envelope from a female $\mathrm{H}$. ismenius (C) Section of signum covered with spines next to a section of the spermatophore envelope from a female E. isabella. Photographs (B) and (C) taken from Galicia, Sánchez \& Cordero (2008) with permission from The Entomological Society of America. 
Spermatophore envelope thickness or Spine length

(median $\pm \mathbf{2 5 \%}$-75\% quartiles) $(\mathrm{mm})$
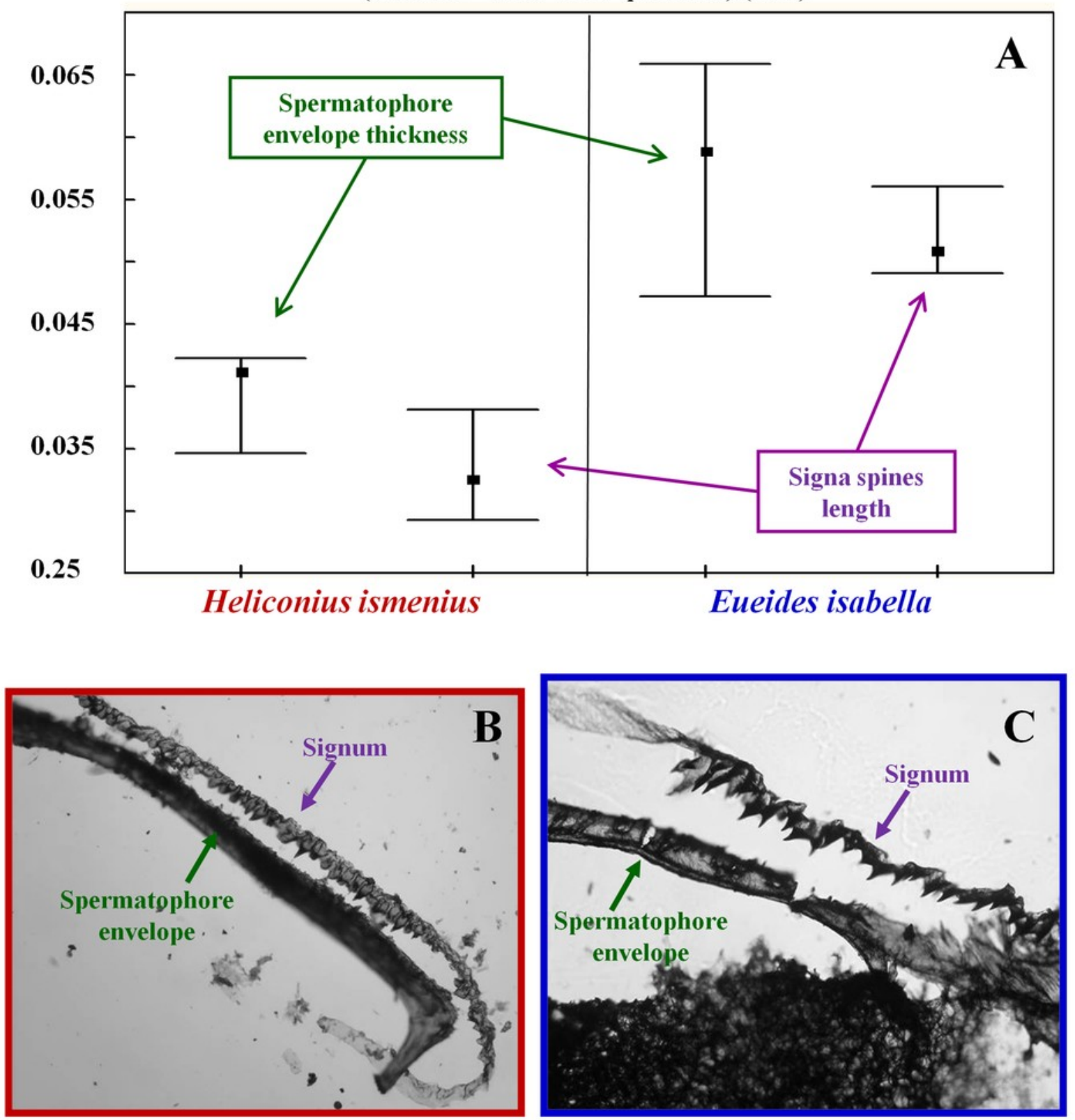


\section{Table 1 (on next page)}

Descriptive statistics of spermatophore envelope thickness $(\mathrm{mm})$ of each spermatophore measured

Each row corresponds to one spermatophore of the species indicated in the first column (total sample: 11 species and 43 spermatophores). ns: total number of measurements made in sections of each individual spermatophore (in almost all cases there were four measurements per section). Species with an asterisk are polyandrous, all the others are monandrous. 


\begin{tabular}{|c|c|c|c|c|c|}
\hline Species/Specimen & $n_{s}$ & Mean & SD & Median & Min.-Max. \\
\hline 1. Heliconius ismenius* & 227 & 0.034 & 0.010 & 0.03 & $0.01-0.06$ \\
\hline 2. H. ismenius* & 157 & 0.035 & 0.012 & 0.03 & $0.01-1.00$ \\
\hline 3. H. ismenius* & 315 & 0.033 & 0.009 & 0.03 & $0.01-0.07$ \\
\hline 4. H. ismenius* & 106 & 0.034 & 0.010 & 0.03 & $0.01-0.06$ \\
\hline 5. H. ismenius* & 154 & 0.037 & 0.010 & 0.04 & $0.02-0.06$ \\
\hline 1. Heliconius hortense & 188 & 0.028 & 0.009 & 0.03 & $0.01-0.05$ \\
\hline 2. H. hortense & 127 & 0.027 & 0.009 & 0.03 & $0.01-0.06$ \\
\hline 3. H. hortense & 179 & 0.028 & 0.008 & 0.03 & $0.01-0.05$ \\
\hline 4. H. hortense & 78 & 0.028 & 0.009 & 0.03 & $0.01-0.04$ \\
\hline 5. H. hortense & 163 & 0.031 & 0.007 & 0.03 & $0.02-0.05$ \\
\hline 1. Heliconius charithonia & 187 & 0.021 & 0.007 & 0.02 & $0.01-0.04$ \\
\hline 2. H. charithonia & 55 & 0.024 & 0.005 & 0.02 & $0.02-0.04$ \\
\hline 1. Eueides aliphera* & 123 & 0.039 & 0.009 & 0.04 & $0.02-0.06$ \\
\hline 2. E. aliphera* & 89 & 0.032 & 0.009 & 0.03 & $0.01-0.06$ \\
\hline 3. E. aliphera* & 102 & 0.036 & 0.008 & 0.04 & $0.02-0.05$ \\
\hline 4. E. aliphera* & 83 & 0.036 & 0.008 & 0.04 & $0.01-0.05$ \\
\hline 1. Eueides isabella & 136 & 0.047 & 0.015 & 0.05 & $0.02-0.09$ \\
\hline 2. E. isabella & 147 & 0.049 & 0.014 & 0.05 & $0.02-0.09$ \\
\hline 3. E. isabella & 232 & 0.060 & 0.018 & 0.06 & $0.02-0.12$ \\
\hline 4. E. isabella & 147 & 0.054 & 0.012 & 0.05 & $0.03-0.10$ \\
\hline 5. E. isabella & 93 & 0.052 & 0.015 & 0.05 & $0.03-0.09$ \\
\hline 6. E. isabella & 209 & 0.052 & 0.015 & 0.05 & $0.03-0.12$ \\
\hline 7. E. isabella & 248 & 0.069 & 0.027 & 0.06 & $0.03-0.16$ \\
\hline 1. Dryadula phaetusa* & 285 & 0.048 & 0.013 & 0.05 & $0.02-0.08$ \\
\hline 2. D. phaetusa* & 221 & 0.045 & 0.011 & 0.05 & $0.02-0.10$ \\
\hline 3. D. phaetusa* & 238 & 0.042 & 0.012 & 0.04 & $0.01-0.08$ \\
\hline 4. D. phaetusa* & 413 & 0.054 & 0.013 & 0.05 & $0.03-0.09$ \\
\hline
\end{tabular}




\begin{tabular}{llllll} 
5. D. phaetusa* & 280 & 0.045 & 0.016 & 0.04 & $0.02-0.11$ \\
1. Dryas iulia* & 236 & 0.047 & 0.014 & 0.05 & $0.01-0.09$ \\
2. D. iulia* $^{*}$ & 195 & 0.033 & 0.012 & 0.03 & $0.01-0.08$ \\
3. D. iulia* & 120 & 0.043 & 0.020 & 0.04 & $0.01-0.09$ \\
1. Philaethria diatonica & 272 & 0.069 & 0.018 & 0.07 & $0.03-0.12$ \\
2. P. diatonica & 333 & 0.070 & 0.018 & 0.07 & $0.03-0.12$ \\
3. P. diatonica & 316 & 0.063 & 0.022 & 0.06 & $0.02-0.21$ \\
1. Agraulis vanillae* & 248 & 0.047 & 0.011 & 0.05 & $0.02-0.08$ \\
2. A. vanillae* & 154 & 0.054 & 0.011 & 0.05 & $0.03-0.09$ \\
3. A. vanillae* & 184 & 0.053 & 0.018 & 0.05 & $0.02-0.14$ \\
1. Dione moneta & 226 & 0.032 & 0.008 & 0.03 & $0.01-0.06$ \\
2. D. moneta & 140 & 0.034 & 0.011 & 0.03 & $0.01-0.08$ \\
3. D. moneta & 219 & 0.037 & 0.013 & 0.04 & $0.01-0.10$ \\
1. Dione juno & 134 & 0.053 & 0.013 & 0.05 & $0.03-0.09$ \\
2. D. juno & 210 & 0.048 & 0.018 & 0.04 & $0.02-0.10$ \\
3. D. juno & 151 & 0.049 & 0.011 & 0.05 & $0.03-0.10$ \\
\hline & & & & &
\end{tabular}

